Saudi Journal of Medical and Pharmaceutical Sciences

Abbreviated Key Title: Saudi J Med Pharm Sci

ISSN 2413-4929 (Print) |ISSN 2413-4910 (Online)

Scholars Middle East Publishers, Dubai, United Arab Emirates

Journal homepage: https://saudijournals.com

Case Report

\title{
Malignant Melanoma of the Nasal Cavity: About 2 Cases
}

El Messaoudi Lina ${ }^{1}{ }^{3 *}$, Bencheikh Razika ${ }^{2},{ }^{3}$, Ahmed Ould Mohamed ${ }^{1},{ }^{3}$, Benbouzid Mohamed Anas ${ }^{2},{ }^{3}$, Leila Essakalli Houssyni $^{2},{ }^{3}$

${ }^{1}$ Resident Physician in Otorhinolaryngology, Department of Otorhinolaryngology, Head and Neck Surgery, Ibn Sina University Hospital, Rabat, Morocco

${ }^{2}$ Professor of Otorhinolaryngology, Department of Otorhinolaryngology, Head and Neck Surgery, Ibn Sina University Hospital, Rabat, Morocco

${ }^{3}$ Faculty of Medicine and Pharmacy of Rabat, Mohammed V University, Rabat, Morocco

DOI: $10.36348 /$ sjmps.2021.v07i01.012

| Received: 04.01.2021 | Accepted: 16.01.2021 | Published: 22.01.2021

*Corresponding author: El Messaoudi Lina

\section{Abstract}

Primitive malignant melanoma of the nasal cavity is a rare tumour of uncertain aetiology and bad prognosis. Unlike skin melanomas, there are no risk factors and the disease is frequently diagnosed in old patients, which clinical complaints are normally non-specific and ranges from nasal obstruction, rhinorrhoea to epistaxis. In most cases this disease is diagnosed in advanced stages which make the treatment difficult.

Keywords: Malignant melanoma, nasal cavity, epistaxis, nasal obstruction, rhinorrhoea.

Copyright $\odot 2021$ The Author(s): This is an open-access article distributed under the terms of the Creative Commons Attribution 4.0 International License (CC BY-NC 4.0) which permits unrestricted use, distribution, and reproduction in any medium for non-commercial use provided the original author and source are credited.

\section{INTRODUCTION}

Malignant melanoma of the nasal cavity is a rare and aggressive tumour, Positive diagnosis of this tumour is made difficult by the non-specific presenting complaints. This tumour has a poor prognosis due to its aggressive nature and the frequently delayed diagnosis. It mainly occurs in the elderly and the presence of comorbidities can limit the extent of treatment. Treatment options essentially consist of radical surgery and radiotherapy, while chemotherapy is reserved for advanced forms. Despite a better knowledge of this tumour, the 5-year overall survival remains poor and does not exceed $40 \%$ in any of the published studies.

\section{Case-1}

A 59-year-old female was admitted in our Department in august 2017 for total left nasal obstruction progressively evolving for about 6 months, recurrent micro epistaxis, without associated ophthalmic signs ; Patient history revealed no systemic diseases and no previous sinonasal surgery .The clinical endoscopic examination found a blackish mucous tumour process of the left nasal fossa filling the nasal floor and the lower and middle meatus and moderate quantity of purulent secretion blood streak in both nostrils with fetid nasal odor ; the cervical palpation found no palpable lymphadenopathy. A tumour biopsy was performed and a CT scan of the nasal sinuses and skull base was done. The biopsy revealed a malignant melanoma and the CT showed a left maxillary nasosinus filling presenting an extension in the nasopharynx with almost total filling of the nasopharyngeal lumen.

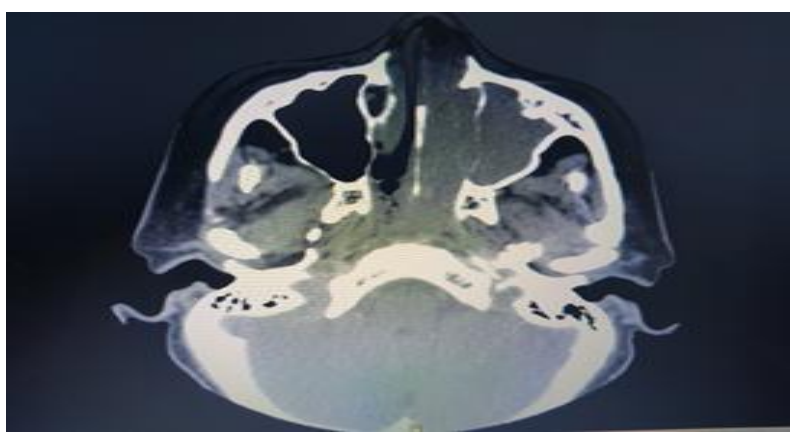

Fig-1: Pre-treatment computed tomography of the facial sinuses. showing a solid voluminous mass extending throughout the left nasal cavity and filling the homolateral maxillary sinus

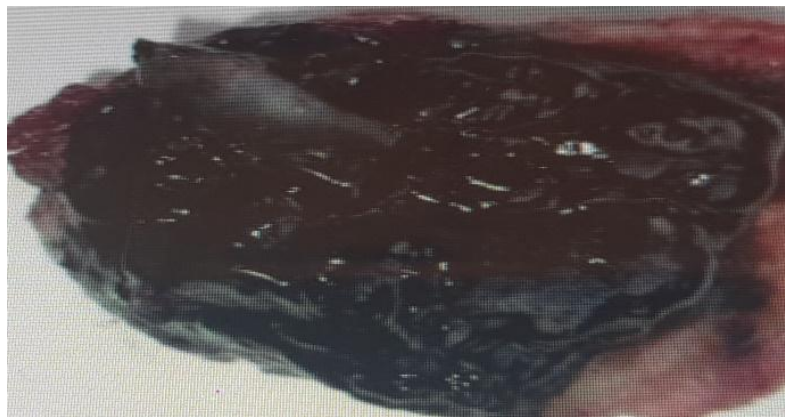

Fig-2: Macroscopic appearance of a malignant melanoma of the nasal cavity 
El Messaoudi Lina et al., Saudi J Med Pharm Sci, Jan, 2021; 7(1): 60-63

The treatment consisted of combined endoscopic endonasal surgery and homolateral Caldwell luck technic for a large resection and an optimal preoperative control with good postoperative improvement. The definitive anatomopathological study of the piece was completed by immunohistochemistry which confirmed the diagnosis of a malignant melanoma with a healthy margin of resection; then the patient was referred to the department of radiotherapy.

\section{Case 2}

40-year-old patient with unilateral left nasal obstruction for one year with fetid rhinorrhoea. The evolution was marked by the installation of a hyposmia with episodes of epistaxis of low abundance with anterior and posterior rhinorrhoea. Nasal endoscopy finds a purplish process originating from the left inferior turbinate which fills the entire nasal cavity preventing visualization of the posterior part. Biological tests, cervical ultrasound, chest X-ray and abdominal ultrasonography were normal. Computer-tomography examination (CT) of naso sinus revealed an expansive process taking the contrast filling the anterior part of the left nasal fossa which caused the nasal obstruction, without osteolysis or cerebral extension.
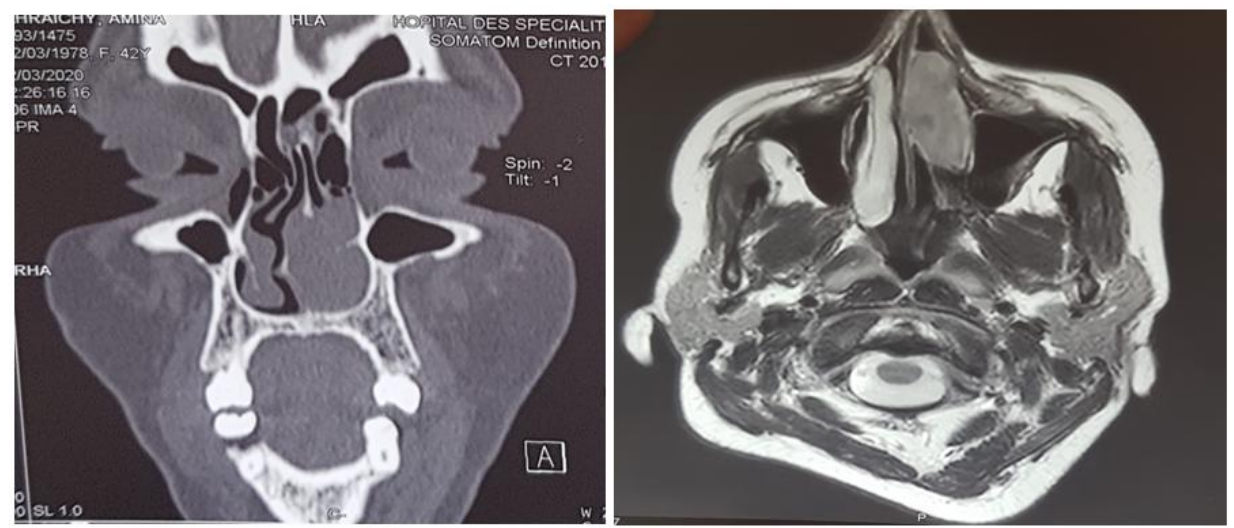

Magnetic resonance imaging and computed tomography of the facial sinuses of the patient showing a tumour of the left nasal cavity with no bone lysis

The neck CT scan did not reveal the presence of lymph nodes. Surgery was based on endoscopic resection of the tumour with lower left turbinectomy. Anatomopathological examination of the surgical piece showed a malignant tumour proliferation of vaguely nodular architecture, within a small intestinal fibroinflammatory stroma. The proliferation consisted of globular cells in the center and spindle-shaped at the periphery. These cells were large, atypical, with a prominent nucleolus and exhibited high mitotic activity. An immunohistichemical study showed clear positivity of the tumour cells to anti-S100, anti-HMB45, antiMelan A and anti- protein antibodies desmin. These histological aspects compared to the immunohistochemical results were in favour of a malignant melanoma. The patient was subsequently referred to the national institute of oncology of Rabat for complementary radiotherapy.

\section{DISCUSSION}

Among the cancers that arise in nasal cavity and par nasal sinuses, mucosal melanomas are rare in comparison to squamous cell carcinomas, it is accounting for $1 \%$ of all systemic malignant melanomas and $4 \%$ of all malignant nasal tumours. It is originates from the dendritic melanocytes of the nasal mucosa that develop from the embryonic neural crest, which belongs to the neuroendocrine cell system [3]. The condition predominantly affects individuals $\geq 60$ years of age; Although possible at any age, it is extremely rare in young people and slightly more common in males [4].The most common sites are the nasal cavity, septum, inferior and middle nasal conchae, the para nasal sinuses are rarely affected but, of these, the maxillary sinus Nasal cavity is more frequently affected than the ethmoid sinus is the most commonly affected [6].

The absence of symptoms in the initial stages delays diagnosis. Furthermore, it is often difficult to differentiate primary melanoma from metastases [1]. The most common initial symptoms are unilateral nasal obstruction and epistaxis; associated symptoms include rhinorrhoea, hyposmia, frontal headache, facial pain, proptosis and diplopia [1, 2]. The prognosis of patients with epistaxis may be better than that of those with nasal obstruction alone [1,3]. Anterior rhinoscopy and fibre optic nasolaryngoscopy may reveal blackish-blue, pale yellow, or in the case of a melanotic melanomas, translucent polypoid masses $[1,6]$. The primary tumour site is often difficult to determine due to the extension of the lesions at diagnosis [2]. In our first case, various structures were affected simultaneously; however the principal site was the right maxillary sinus, thus characterizing the primary site of the lesion [9]. When satellite lesions are present and there are concomitant area of amelanotic melanoma, the case becomes more complicated $[3,5]$. 
El Messaoudi Lina et al., Saudi J Med Pharm Sci, Jan, 2021; 7(1): 60-63

The diagnosis is based on histological examination of tumour biopsies. Histological examination is difficult due to marked cytological and architectural polymorphism. Confirmation of diagnosis is based on immunohistochemistry using a panel of markers: tyrosinase (the most sensitive marker for nasosinus melanoma), Mart-1 / Melan-A, anti-gp100 (HMB-45), protein S100, Microphthalmia transcription factor (MIFT), or even PNL-2 [1, 2].

An imaging assessment comprising computed tomography (CT) of the facial bones and magnetic resonance imaging (MRI) is the most efficient examination for specifying local extension in the sinus and extranasosinus cavities, extension to the orbit, infra-temporal fossa and cavernous sinus [2, 8]. For extension to the soft tissues of the face, computed tomography remains quite effective, but MRI allows a more detailed study. The general workup for the disease includes a chest $\mathrm{x}$-ray, abdomino pelvic ultrasound and bone scan [1].

The differential diagnosis of a nasosinusal tumour includes various round cell tumours, which may be benign, intermediate and malignant. Benign tumours include nasal polyps, osteoma, chondroma, schwannoma and neurofibroma, cementoma. Tumours classified as intermediate include inverted papillomas, meningiomas, hemangioma and hemangiopericytomas, and of the malignant one, squamous cell carcinoma is the most common, followed by adenoid cystic carcinoma, adenocarcinoma, neuroblastoma, "rotundcell" sarcoma, lymphoma and undifferentiated carcinoma with small cells [4].

The clinical and imaging assessments allows Staging of the melanoma in order to propose Adapted treatment and to assess the prognosis. Ballantyne's classification is the oldest classification, but does not take tumour size tumour Histology and local extension into account [11]. As stage 1 is defined as tumour confined to the original site. Stage 2 is defined as tumour with regional lymph node metastases and stage 3 is defined as tumour with distant metastases. Use of this old classification allows comparison of various series. But it is now preferable to refer to the classification established by the American joint committee on Cancer. The 7th edition of the AJCC classification does not comprise stage $\mathrm{T} 1$ and $\mathrm{T} 2$ in view of the systemically aggressive nature of this melanomas [7, 10]. The proposed classification comprising stage T3 and T4 tumours is more consistent with the local extension and the poor prognosis of this disease [8]. First-line treatment consists of surgical resection, Prophylactic neck nodal dissection at N0 is not recommended, since the incidence of occult nodal metastases is relatively low [3]. Local recurrence occurs in $29-79 \%$ of cases despite aggressive surgery and may be clinically devastating $[3,4]$. Some authors recommend radiotherapy for local control, while others have reported no improvement in overall survival. Chemotherapy, most commonly with actinomycin D, interferon, and cisplatin, is used at more advanced stages, generally for palliative purposes or when surgery is contraindicated [5]. In addition to interferon, interleukin 2 and bacillus Calmette-Guerin are other possible options for immunotherapy; Surgery may be an option later. For patients with sinonasal melanoma, prognosis is poor, sine this tumour is aggressive and normally diagnosed at more advanced stages. More than $50 \%$ of patients die within three years of diagnosis, some within a few weeks or months of developing symptoms, with the disease disseminating rapidly despite surgical treatment[]. Here, the disease was advanced at diagnosis; however, the structures of the central nervous system were not affected $[2,3]$.

\section{CONCLUSION}

Despite the rarity, melanoma of the nasal cavities requires special attention given its poor prognosis. Therefore, it appears essential to perform a careful rhinoscopic examination in the face of any lingering sinus symptomatology, unilateral symptoms such as epistaxis and nasal obstruction especially in a patient over the age of 60 years must be considered to be suspicions, the diagnosis is based on histological examination of tumour biopsies and Surgery remains the first-line treatment, radiotherapy as an adjuvant treatment, seems to improve local control according to some studios,chemotherapy is useful in the palliative context, the overall the prognosis of this tumours is very poor; hopes for improvement of survival are based in progress of radiotherapy modalities and gene therapy that are under evaluation.

\section{REFERENCES}

1. Alves, I. S. S., Berriel, L. G. S., Alves, R. T., Pinto, M. B., Oliveira, C. F. P., Cazzotto, A. C., \& Moura, W. V. (2017). Sinonasal melanoma: a case report and literature review. Case Reports in Oncological Medicine, 2017.

2. Kingdom, T. T., \& Kaplan, M. J. (1995). Mucosal melanoma of the nasal cavity and paranasal sinuses. Head \& neck, 17(3), 184-189.

3. Dwivedi, R., Dwivedi, R., Kazi, R., Kumar, S., \& Agarwal, S. P. (2008). Mucosal melanoma of nasal cavity and paranasal sinus. Journal of cancer research and therapeutics, 4(4), 200.

4. Melanoma: clinical review of 23 cases, Journal of the Chines Medical Association. 24-29, 2007.

5. Marcus, D. M., Marcus, R. P., Prabhu, R. S., Owonikoko, T. K., Lawson, D. H., Switchenko, J., \& Beitler, J. J. (2012). Rising incidence of mucosal melanoma of the head and neck in the United States. Journal of skin cancer, 2012.

6. Nakaya, M., Mochiki, M., Takeuchi, S., Yuge, T., Nakao, K., Nakamura, N., \& Sugasawa, M. 
(2004). Malignant melanoma of nasal cavity: report of 16 Japanese patients. Auris Nasus Larynx, 31(3), 233-237.

7. Rekik, W., Goucha, A., Hamdane, M., Debbabi, B., El May, A., \& Gammoudi, A. (2012). Malignant melanoma of the nasal cavity. Pan Afr Med J. 12:6

8. Kharoubi, S. (2005). Malignant melanoma of nasal fossae: clinical and therapeutic considerations about three cases. Cancer radiotherapie: journal de la Societe francaise de radiotherapie oncologique, 9(2), 99-103.

9. Luna-Ortiz, K., Aguilar-Romero, M., Villavicencio-Valencia, V., Zepeda-Castilla, E., Vidrio-Morgado, H., Peteuil, N., \& MosquedaTaylor, A. (2016). Comparative study between two different staging systems (AJCC TNM VS BALLANTYNE'S) for mucosal melanomas of the Head \& Neck. Medicina Oral, Patología Oral y Cirugía Bucal, 21(4), e425.

10. Patrick, R. J., Fenske, N. A., \& Messina, J. L. (2007). Primary mucosal melanoma. Journal of the American Academy of Dermatology, 56(5), 828-834.

11. Castillo, L., Poissonnet, G., Haddad, A., Dassonville, O., \& Santini, J. (1998). Mélanomes malins naso-sinusiens. Bilan et stratégie thérapeutique. Les Cahiers d'oto-rhinolaryngologie, de chirurgie cervico-faciale et d'audiophonologie, 33(8), 426-432.

12. Kardon, D. E., \& Thompson, L. D. (2000). Sinonasal mucosal malignant melanoma: report of an unusual case mimicking schwannoma. Annals of Diagnostic Pathology, 4(5), 303-307.

13. Lee, S. P., Shimizu, K. T., Tran, L. M., Juillard, G., \& Calcaterra, T. C. (1994). Mucosal melanoma of the head and neck: the impact of local control on survival. The Laryngoscope, 104(2), 121-126.

14. Brożyna, A. A., Jóźwicki, W., Roszkowski, K., Filipiak, J., \& Slominski, A. T. (2016). Melanin content in melanoma metastases affects the outcome of radiotherapy. Oncotarget, 7(14), 17844.

15. Mendenhall, W. M., Amdur, R. J., Hinerman, R.
W., Werning, J. W., Villaret, D. B., \& Mendenhall, N. P. (2005). Head and neck mucosal melanoma. American journal of clinical oncology, 28(6), 626-630.

16. Bouguila, J., Zairi, I., Jablaoui, Y., Haddad, S., Hellali, M., Azzouz, E., ... \& Adouani, A. (2006). Mélanome malin des fosses nasales révélé par une tuméfaction jugale. Revue de Stomatologie et de Chirurgie Maxillo-faciale, 107(6), 474-476.

17. Manolidis, S., \& Donald, P. J. (1997). Malignant mucosal melanoma of the head and neck: review of the literature and report of 14 patients. Cancer: Interdisciplinary International Journal of the American Cancer Society, 80(8), 1373-1386.

18. Chiu, N. T., \& Weinstock, M. A. (1996). Melanoma of oronasal mucosa: population-based analysis of occurrence and mortality. Archives of Otolaryngology-Head \& Neck Surgery, 122(9), 985-988.

19. Garzino-Demo, P., Fasolis, M., Maggiore, G. M. L. T., Pagano, M., \& Berrone, S. (2004). Oral mucosal melanoma: a series of case reports. Journal of Cranio-Maxillofacial Surgery, 32(4), 251-257.

20. Yii, N. W., Eisen, T., Nicolson, M., A'Hern, R., Rhys-Evans, P., Archer, D., ... \& Gore, M. E. (2003). Mucosal malignant melanoma of the head and neck: the Marsden experience over half a century. Clinical oncology, 15(4), 199-204.

21. Going J. J., \& Kean, D. M. (1989). Malignant melanoma of the nasal cavity. J Laryngol Otol. 103:231-3.

22. Hoyt, D. J., Jordan, T., \& Fisher, S. R. (1989). Mucosal melanoma of the head and neck. Archives of Otolaryngology-Head \& Neck Surgery, 115(9), 1096-1099.

23. Ordonez, N. G., Sneige, N., Hickey, R. C., \& Brooks, T. E. (1988). Use of monoclonal antibody HMB-45 in the cytologic diagnosis of melanoma. Acta cytologica, 32(5), 684-688.

24. Dauer, E. H., Lewis, J. E., Rohlinger, A. L., Weaver, A. L., \& Olsen, K. D. (2008). Sinonasal melanoma: a clinicopathologic review of 61 cases. Otolaryngology-Head and Neck Surgery, 138(3), 347-352. 